\title{
High efficiency, multiterawatt $x$-ray free electron lasers
}

\author{
C. Emma, ${ }^{1}$ K. Fang, ${ }^{2}$ J. Wu, ${ }^{2}$ and C. Pellegrini ${ }^{1,2}$ \\ ${ }^{1}$ University of California, Los Angeles, California 90095, USA \\ ${ }^{2}$ Stanford Linear Accelerator Center, Menlo Park, California 94025, USA
}

(Received 28 October 2015; published 26 February 2016)

\begin{abstract}
In this paper we present undulator magnet tapering methods for obtaining high efficiency and multiterawatt peak powers in x-ray free electron lasers (XFELs), a key requirement for enabling 3D atomic resolution single molecule imaging and nonlinear $\mathrm{x}$-ray science. The peak power and efficiency of tapered XFELs is sensitive to time dependent effects, like synchrotron sideband growth. To analyze this dependence in detail we perform a comparative numerical optimization for the undulator magnetic field tapering profile including and intentionally disabling these effects. We show that the solution for the magnetic field taper profile obtained from time independent optimization does not yield the highest extraction efficiency when time dependent effects are included. Our comparative optimization is performed for a novel undulator designed specifically to obtain TW power x-ray pulses in the shortest distance: superconducting, helical, with short period and built-in strong focusing. This design reduces the length of the breaks between modules, decreasing diffraction effects, and allows using a stronger transverse electron focusing. Both effects reduce the gain length and the overall undulator length. We determine that after a fully time dependent optimization of a $100 \mathrm{~m}$ long Linac coherent light source-like XFEL we can obtain a maximum efficiency of 7\%, corresponding to $3.7 \mathrm{TW}$ peak radiation power. Possible methods to suppress the synchrotron sidebands, and further enhance the FEL peak power, up to about 6 TW by increasing the seed power and reducing the electron beam energy spread, are also discussed.
\end{abstract}

DOI: 10.1103/PhysRevAccelBeams.19.020705

\section{INTRODUCTION}

$\mathrm{X}$-ray free electron lasers (XFELs) are tunable and highly versatile light sources, capable of generating coherent radiation over a very broad spectral range. From tens of nanometers to a fraction of 1 angstrom, the peak power is in the range of 10 to about $50 \mathrm{GW}$ and the pulse duration from a few to about 100 femtoseconds [1,2]. This versatility has rendered XFELs highly successful in a wide range of scientific applications. Among these are nonlinear $\mathrm{x}$-ray physics and the determination of structural information from biological samples, with recent experiments achieving angstrom level spatial and femtosecond time-scale resolution using $\mathrm{x}$-ray diffraction imaging techniques. One of the major goals for future XFELs is to further increase the resolution of these experiments in order to enable 3D atomic resolution imaging of single molecules while maintaining a short enough pulse duration to limit radiation damage on the sample [3]. Recent unexpected results on nonlinear Compton scattering also show the interest in increasing the peak power level and bring the electric field in the focused x-ray beam nearer to the Schwinger critical

Published by the American Physical Society under the terms of the Creative Commons Attribution 3.0 License. Further distribution of this work must maintain attribution to the author(s) and the published article's title, journal citation, and DOI. field [4]. Both these applications require x-ray pulses with peak powers in the terawatt (TW) range.

An important parameter for FELs is the efficiency, the ratio of the FEL radiation power to the electron beam power. In the $\mathrm{x}$-ray region the FEL efficiency is rather low, about $10^{-3}$ at saturation. Increasing the efficiency beyond the saturation value is possible by tapering the undulator magnetic field [5]. This has been examined theoretically $[5,6]$, numerically [7-9] and demonstrated experimentally from the microwave [10] down to hard X-ray wavelengths [11]. At hard X-ray wavelengths the achieved efficiencies are a factor of 3 larger than the exponential saturation value [11]. Despite this substantial improvement the peak power levels at state of the art facilities are still insufficient for single molecule imaging and nonlinear science applications which require an efficiency increase of more than an order of magnitude [3]. This has sparked renewed interest in the community with a number of recent studies specifically devoted to finding the optimal tapering law using a model-based approach and a form of parametric optimization $[8,9,12]$. With the exception of Ref. [12], which considers longer wavelengths than we do in this paper, the optimizations presented have dealt exclusively with the time independent physics of tapered FELs, producing a taper profile which maximizes the extraction efficiency ignoring time dependent effects like the synchrotron sideband instability [5].

The purpose of this paper is to analyze the impact of time dependent effects on the optimization of tapered hard $\mathrm{x}$-ray 
FEL amplifiers. We present the first detailed comparison of time independent and time dependent optimizations and discuss the limits set on the efficiency by time dependent processes like the synchrotron sideband instability. We show that the solution obtained for the optimal taper profile in time independent simulations does not yield the maximum extraction efficiency when fully time dependent physics is included in the dynamics of the electron beam and radiation field system. We also analyze the FEL interaction upstream of the tapered undulator and highlight the importance of the trade-off between input seed power and energy spread in a tapered self-seeded system.

We study the optimization problem by following the multidimensional scan method of Ref. [7] for a superconducting, $2 \mathrm{~cm}$ period helical undulator with built-in focusing. This novel undulator design is optimized for maximum efficiency, reduction of intramodule undulator length, strong transverse focusing, short gain length and minimum total undulator length. While we do not intend to present a full engineering design of the undulator in this study, feasibility considerations are made with regard to realizing such an undulator in practice.

The paper is divided as follows. In Sec. II we discuss the undulator design, outlining the undulator parameters and the feasibility of practical realization. In Sec. III we discuss the self-seeding method and simulate the self-amplified spontaneous emission (SASE) FEL [13] upstream of the tapered undulator. In Secs. IVA and IV B we present the time independent and time dependent tapering optimization results and discuss the differences between the two. In Sec. V we analyze in detail the sideband instability, its onset, amplification and its impact on particle dynamics and taper saturation. Finally we discuss methods to suppress the instability and outline future work on high efficiency TW level XFELs.

\section{UNDULATOR DESIGN}

We apply the tapering optimization method [7] to an undulator designed specifically to achieve TW power x-ray pulses in the shortest possible undulator length. Our ideal undulator is superconducting, with a short $2 \mathrm{~cm}$ period and a peak on axis field $B_{0}$ of $1.6 \mathrm{kG}$. For a double helix bifilar magnet with equal and opposite currents this field is given by [14]

$$
B_{0}=\frac{4 k_{u} I}{10}\left[k_{u} a K_{0}\left(k_{u} a\right)+K_{1}\left(k_{u} a\right)\right],
$$

where $B_{0}$ is the field in Gauss, $I$ is the current in Ampère, $k_{u}=2 \pi / \lambda_{u}$ is the undulator wave number, $a$ is the helix radius and $K_{0}$ and $K_{1}$ are modified Bessel functions. For a helical bore radius $a=7.5 \mathrm{~mm}$ the total current required through the coils is $I=484 \mathrm{~A}$, which, considering coils of $\sim \mathrm{mm}^{2}$ surface area, gives a current density below the critical value for superconducting $\mathrm{NbTi}$ or $\mathrm{Nb3Sn}$ wires.
From the point of view of operation a superconducting undulator has advantages such as resistance to radiation damage and reduced sensitivity to wakefields, for a more detailed description see Ref. [15]. The undulator is designed to be helically polarized as this increases the effect of refractive guiding in the postsaturation regime and improves the FEL performance [16].

In order to accommodate diagnostics a realistic undulator design must include periodic break sections, with longer breaks adversely affecting performance. This is due essentially to three effects. First, diffraction effects are critical to the performance of a tapered FEL, particularly for long, multiple Rayleigh length undulators. While these effects are mitigated by refractive guiding inside the undulator, there is no guiding during the break sections and the radiation size increases, reducing the field amplitude, causing particle detrapping and limiting the extraction efficiency. Second, a break of length $L_{b}$ introduces a phase error $\Delta \Psi \sim$ $L_{b} \delta / \gamma^{2}=2 n \lambda_{r} \eta$ for a particle with relative energy offset $\eta=\delta \gamma / \gamma_{r}$ with respect to the resonant particle. Thus longer break sections increase electron phase mixing and reduce the bunching factor. Finally as a practical consideration, for a given total undulator length, longer break sections reduce the length of magnetic elements, limiting the electron deceleration and overall extraction efficiency. To minimize the break length we superimpose the focusing quadrupole field on the helical undulator field, similar to the design successfully tested in Ref. [17]. One advantage of distributed quadrupole focusing is the possibility of operating at small betatron beta function, due to the reduced FODO lattice length $L_{f}$. This minimizes the transverse beam envelope oscillation $\Delta \beta^{2} / \beta_{a v}^{2}=\beta_{a v} L_{f} /\left(\beta_{a v}^{2}-L_{f}^{2}\right)$ which also degrades the FEL performance [18]. In our study the undulator magnetic field is tapered continuously and the section length is chosen to be $1 \mathrm{~m}$, close to the 3D gain length with $20 \mathrm{~cm}$ breaks in between. We note that a stepwise taper profile for short section lengths on the order of a gain length does not critically affect the performance, as discussed in detail in Ref. [19].

Although this kind of undulator has never been constructed in the past, the parameters presented in this design are similar to what is currently being considered for an Linac coherent light source (LCLS)-II-like planar superconducting undulator with the addition of built-in quadrupole focusing [15]. Integrating quadrupoles in the design presents several technical challenges. For example it sets a more stringent requirement on alignment tolerances. A full engineering and tolerance study of this undulator is needed before we can be confident that it is a feasible option for future high efficiency x-ray FEL facilities.

\section{SELF-SEEDING STAGE}

We consider generating a monochromatic seed pulse using self-seeding design based on a single crystal 


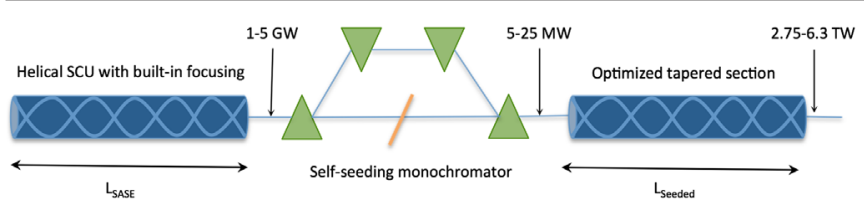

FIG. 1. Schematic of the undulator for hard X-ray multi-TW peak power output, designed to achieve high extraction efficiency in the shortest possible distance.

monochromator, similar to what is currently installed at LCLS [20]. We assume that the tapered section follows a self-seeding chicane which completely eliminates the beam microbunching, and is preceded by a SASE section as shown in Fig. 1. The energy spread at the start of the tapered section is determined by the SASE FEL process, spontaneous emission losses in the SASE section and the laser heater induced energy spread set to suppress the microbunching instability [21,22]. We perform a simulation of the SASE section assuming an initial rms energy spread due to the linac of $10^{-4}$. The growth of the SASE power and rms energy spread are shown in Fig. 2.

For effective seeding, the radiation power must exceed the electron beam shot noise power $[23,24]$ :

$$
P_{\text {noise }} \approx \gamma m c^{2} \omega_{r} \rho^{2} / 2
$$

by a wide margin. For our parameters this evaluates to $36 \mathrm{~kW}$. We therefore decide to start the seeded section in our baseline case with $5 \mathrm{MW}$ of power, which requires $1 \mathrm{GW}$ of SASE power incident on the monochromator given a seeding efficiency of $0.5 \%$ as in the LCLS [20]. This sets the length of the SASE section $L_{\mathrm{SASE}}=13.4 \mathrm{~m}$ and the input energy spread $\sigma_{E}=3.1 \mathrm{MeV}$. As pointed out in Ref. [20], in practice there is a trade-off between seed power and energy spread at the start of the seeded section. To analyze how this trade-off impacts the tapered FEL performance we study different cases for input seed powers of 5 and $25 \mathrm{MW}$ and energy spreads of $\sigma_{E}=3.1 \mathrm{MeV}$ and

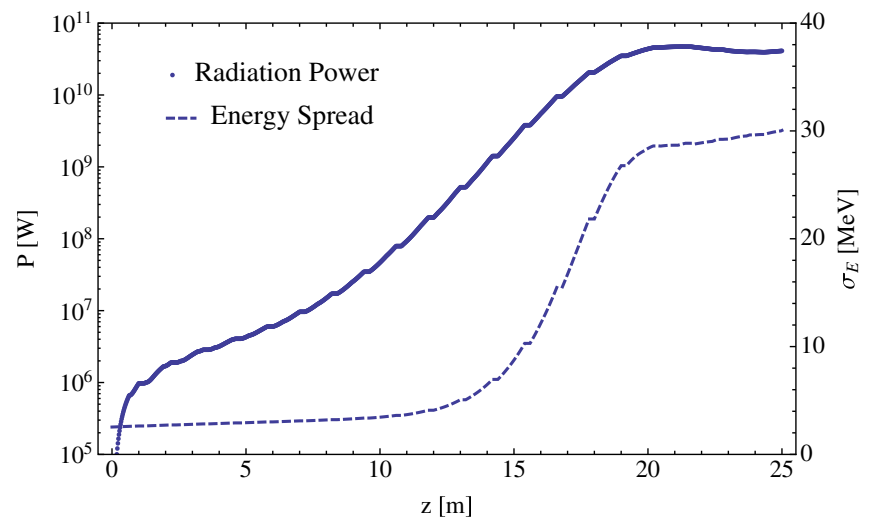

FIG. 2. SASE power and energy spread upstream of the selfseeding chicane.
$\sigma_{E}=1.5 \mathrm{MeV}$. The two cases with $1.5 \mathrm{MeV}$ energy spread are representative of a fresh bunch configuration in which the beam lasing in the seeded section accumulates no additional energy spread in the SASE section. Methods for achieving such a configuration are described in the following sections.

\section{TAPERING OPTIMIZATION}

\section{A. Time independent}

We first obtain the optimal taper profile, maximizing the output power for a fixed $100 \mathrm{~m}$ undulator length in time independent simulations using the three-dimensional FEL particle code GENESIS [25]. The tapering law is written as

$$
a_{w}(z)= \begin{cases}a_{w 0}, & z<z_{0} \\ a_{w 0} \times\left(1-c \times\left(z-z_{0}\right)^{d}\right), & z>z_{0}\end{cases}
$$

where the parameters $z_{0}, c, d$ are obtained by multidimensional scans that maximize the output radiation power. The simulation parameters are given in Table I and are all performed for a transversely flat electron beam distribution as this maximizes the output power, as described in Ref. [26]. The quadrupole focusing can also be tapered to further increase the extraction efficiency as shown in Ref. [7] but that will not be considered in this study. The optimal taper profile obtained from time independent optimization is shown in Fig. 3. The tapering order is approximately quadratic, which follows qualitatively from the fact that in time independent simulations the bunching factor and trapping fraction remain nearly constant in the tapered section, and the dominant radiative process is coherent emission. The peak output power is $7.3 \mathrm{TW}$ with an extraction efficiency of $14 \%$. It is important to note that there is little sign of the taper power saturating in the time

TABLE I. GENESIS Simulation parameters.

\begin{tabular}{lr}
\hline \hline Parameter name & Parameter value \\
\hline Beam energy & $12.975 \mathrm{GeV}$ \\
Peak current & $4000 \mathrm{~A}$ \\
Normalized emittances & $0.3 / 0.3 \mu \mathrm{m} \mathrm{rad}$ \\
Average beta function & $5 \mathrm{~m}$ \\
rms energy spread & $10^{-4}$ \\
Bunch length & $24 \mathrm{fs}$ \\
Seed radiation power & $5-25 \mathrm{MW}$ \\
Radiation wavelength & $1.5 \AA$ \\
Rayleigh length & $10 \mathrm{~m}$ \\
Undulator period & $2 \mathrm{~cm}$ \\
Undulator parameter & 3 \\
Quadrupole focusing strength & $26.4 \mathrm{~T} / \mathrm{m}$ \\
Undulator section length & $1 \mathrm{~m}$ \\
Undulator break length & $20 \mathrm{~cm}$ \\
FEL parameter & $1.66 \times 10^{-3}$ \\
3D gain length & $65 \mathrm{~cm}$ \\
\hline \hline
\end{tabular}



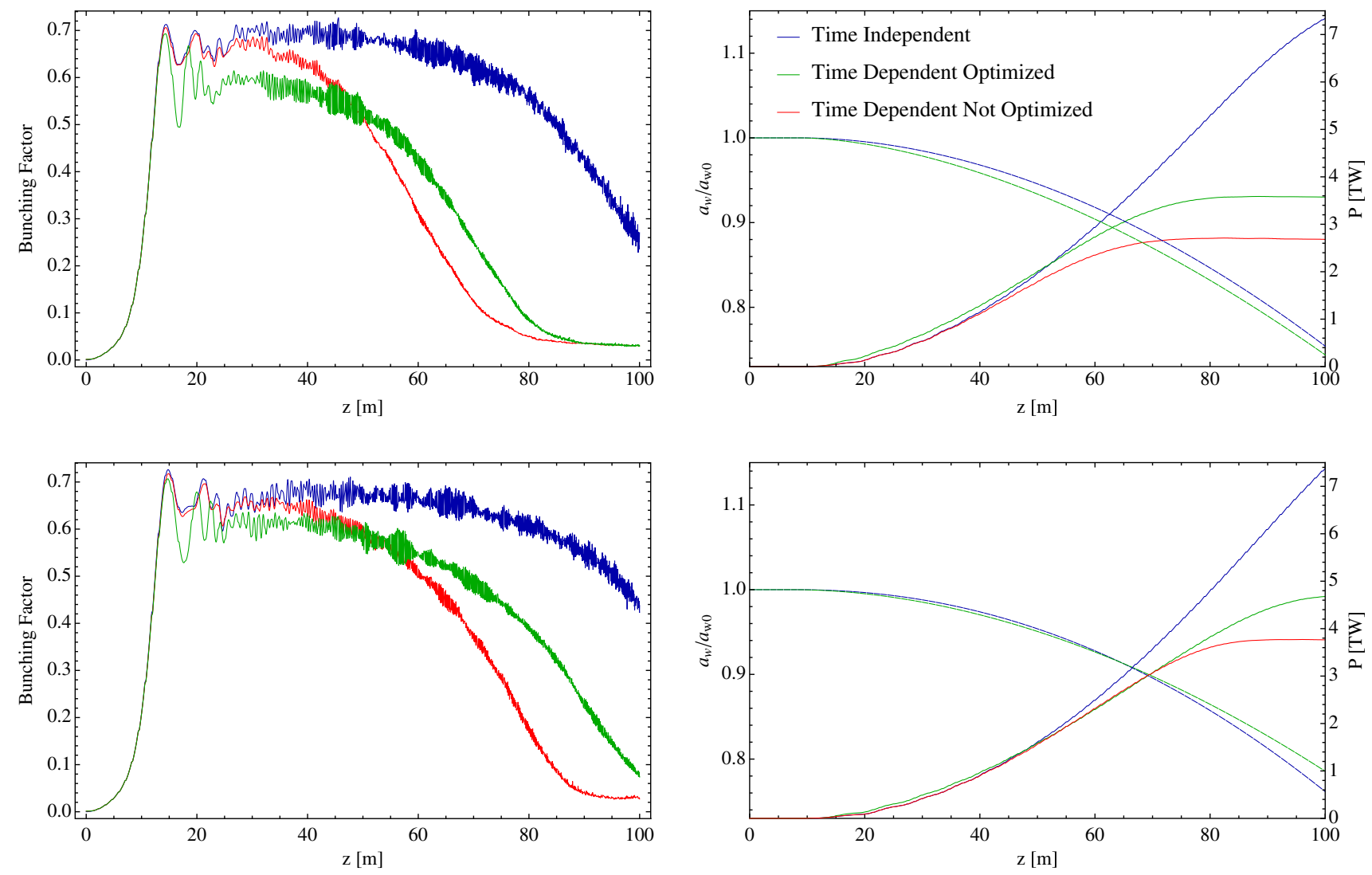

FIG. 3. Bunching factor (left), taper profiles and FEL radiation power evolution (right) obtained from time independent and time dependent optimization. The top plots correspond to an input energy spread $\sigma_{E, 0}=3.1 \mathrm{MeV}$ consistent with the SASE result from Fig. 1. The bottom plots are an alternate case with $\sigma_{E, 0}=1.5 \mathrm{MeV}$. In both cases $z=0$ is after the self-seeding monochromator and the input seed power is $5 \mathrm{MW}$.

independent case, which is not the case when time dependent effects are included.

\section{B. Time dependent optimization}

The physical difference between time independent and time dependent optimization arises from noise in the electron beam current distribution and slippage of the radiation field. This can drive the amplification of parasitic frequencies and the sideband instability, causing temporal fluctuations in the electric field profile, particle detrapping and eventually taper saturation.

Using the optimal taper starting point obtained from time independent simulations, we perform time dependent scans over the taper order $d$ and the taper strength $\Delta a_{w} / a_{w}=$ $c \times\left(L_{w}-z_{0}\right)^{d}$. As shown in Figs. 3 and 4 , the values of the taper order and taper strength yielding the maximum power in time independent simulations are not the optimal choice of parameters once time dependent effects are included. The variation in peak power is more sensitive to variations in the taper profile in the time dependent cases. The optimal taper order is weaker than quadratic, and is reduced compared to the time independent case. This is due to the FEL's increased sensitivity to particle detrapping when electron beam shot noise and multiple frequency effects are included.

Since the coherent emission power is proportional to the product of the number of trapped particles and the change in resonant energy (taper strength), a slower taper preserves the trapping for longer, maximizing the product and the overall extraction efficiency. In the large energy spread case it is important to note that the time dependent optimized taper profile has a slower taper order but a larger overall deceleration rate. This results in a worse particle capture in the early stages of the tapered section $(z=10-50 \mathrm{~m})$ but a reduction in detrapping in the remainder of the undulator. This can be understood by examining the functional form for the resonant phase which determines the bucket area and the bucket height:

$$
\sin \Psi_{R}(z)=\frac{\lambda_{w}}{4 \pi} \frac{\left|a_{w}^{\prime}(z)\right|}{a_{s}(z)},
$$

where $a_{s}=e A_{s} / m_{e} c^{2}$ is the dimensionless vector potential for the electric field. The time dependent optimized taper reduces $\left|a_{w}^{\prime}(z)\right|$ in the second half of the undulator $z=50-100 \mathrm{~m}$. This maintains a larger bucket area in 

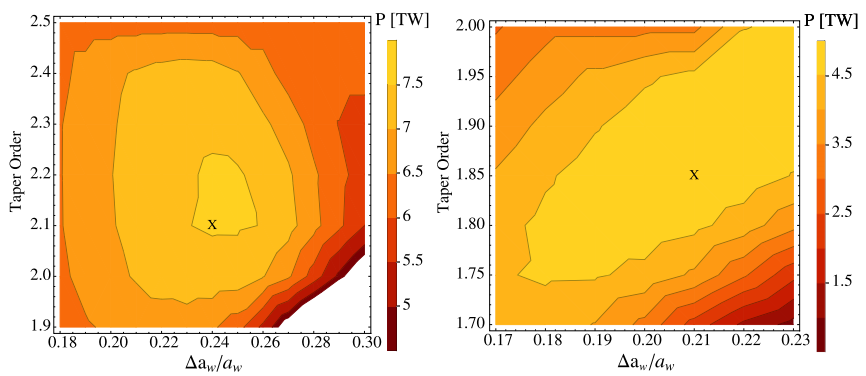

FIG. 4. Maximum radiation power as a function of taper order $d$ and taper amplitude $\Delta a_{w} / a_{w}$ for time independent (left) and time dependent (right) simulations for the case of $1.5 \mathrm{MeV}$ input energy spread.

the region where the amplitude of the sidebands is more appreciable and the system is more sensitive to sideband induced detrapping. In an ideal system, the taper profile would have an improved capture rate in the early stages, with a profile similar to what one obtains from time independent optimization. In the later stages, when time dependent effects are more appreciable, the taper profile should have a slower decrease in the undulator field similar to what one obtains from time dependent optimization. This requires a more elaborate piecewise functional form for $a_{w}(z)$ and will be investigated in future work.

\section{Effect of the energy spread}

The input energy spread is a critical parameter for the performance of a tapered x-ray FEL. We study this by performing the same time dependent optimization for two cases both starting with a $5 \mathrm{MW}$ seed: the self-seeded case with an energy spread of $3.1 \mathrm{MeV}$ and an alternate case with 1.5 MeV. In practice, the alternate case could be achieved by considering a double-bunch system, where two closely spaced bunches are separated in time before the entrance to the undulator. The first bunch is sent through the undulator to lase producing the seed radiation and is discarded prior to the self-seeding chicane. The trailing bunch lasing is deliberately suppressed in the SASE section, for example by inducing betatron oscillations, and is recombined with the seed pulse with a corrected orbit downstream of the self-seeding chicane. In this scheme the seeded bunch would have an rms energy spread set only by the linac and the laser heater, around $1.5 \mathrm{MeV}$ for our beam parameters.

As is evidenced in Fig. 3, the low energy spread case achieves a higher peak power, $4.7 \mathrm{TW}$ compared to $3.7 \mathrm{TW}$, after the time dependent optimization. The taper saturation is also delayed due to a decrease in sideband induced particle detrapping. In both cases electron emission into the lower synchrotron sideband mode causes detrapping from the high energy region of the stable phase space area. Furthermore, scattering of the electrons from interaction with the sideband frequencies causes diffusion and additional particle loss. This is illustrated in Fig. 5 where the

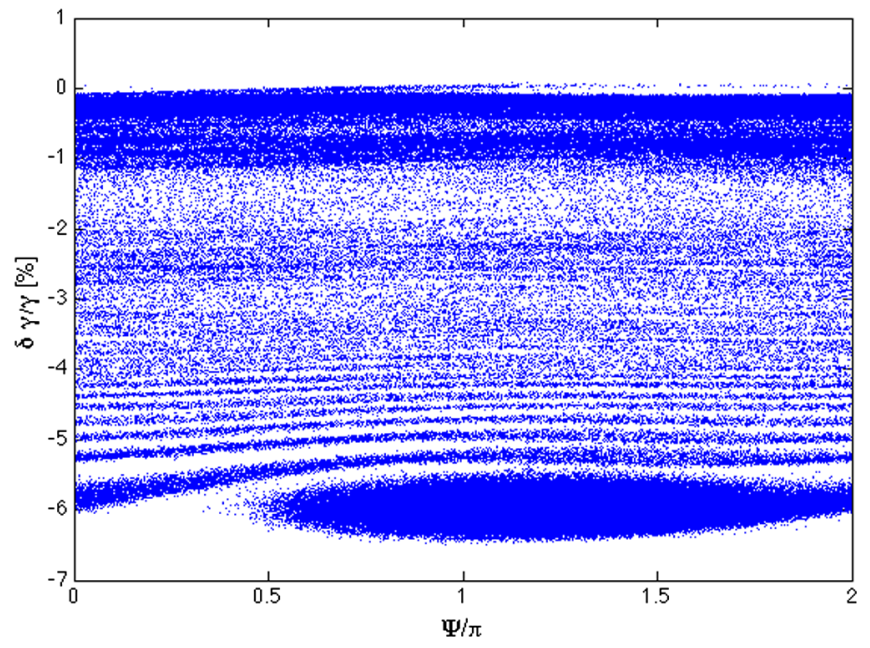

FIG. 5. Longitudinal phase space for the core 6 fs of the beam at the undulator exit in the $3.1 \mathrm{MeV}$ energy spread, $5 \mathrm{MW}$ seed case. Particle detrapping in the second half of the undulator is caused by the synchrotron sideband instability. Note that multiple phase spaces are overlapped within one wavelength in the $0<\Psi<2 \pi$ range.

longitudinal phase space for the core 6 fs of the beam in the 3.1 MeV optimized case is plotted at the undulator exit. In the next section we examine the effects of sidebandinduced detrapping and show how the time dependent optimization reduces them.

\section{SIDEBAND INSTABILITY}

The mechanism of sideband generation and amplification in free electron lasers can be summarized as follows [27]. First, sidebands are generated due to amplitude and phase modulations of the electric field which result from the trapped particles undergoing synchrotron oscillations as they pass through the undulator. Using Maxwell's equations in the 1D slowly varying envelope approximation [27] we can write the evolution of the electric field amplitude $a_{s}$ and phase variation $\delta k_{s}=k_{s}-\omega_{s} / c$ as a function of the undulator distance $z$ and the intrabunch coordinate $s$ :

$$
\begin{gathered}
a_{s}^{\prime}(s, z)=\frac{\omega_{p}^{2}(s, z)}{2 \omega_{s} c} a_{w}(z)\left\langle\frac{\sin \Psi(s, z)}{\gamma(s, z)}\right\rangle \\
\delta k_{s}(s, z)=\frac{\omega_{p}^{2}(s, z)}{2 \omega_{s} c} \frac{a_{w}(z)}{a_{s}(s, z)}\left\langle\frac{\cos \Psi(s, z)}{\gamma(s, z)}\right\rangle,
\end{gathered}
$$

where $\omega_{p}$ is the electron beam plasma frequency and $\Psi$ is the ponderomotive phase. It is clear from these that as the electrons oscillate in the longitudinal phase space $(\Psi, \gamma)$ the gain and the phase shift of the radiation field will be different at different locations in the undulator and, due to shot noise in the electron beam, at different locations along the bunch. This results in a temporal modulation of the radiation field giving rise to sidebands displaced from 

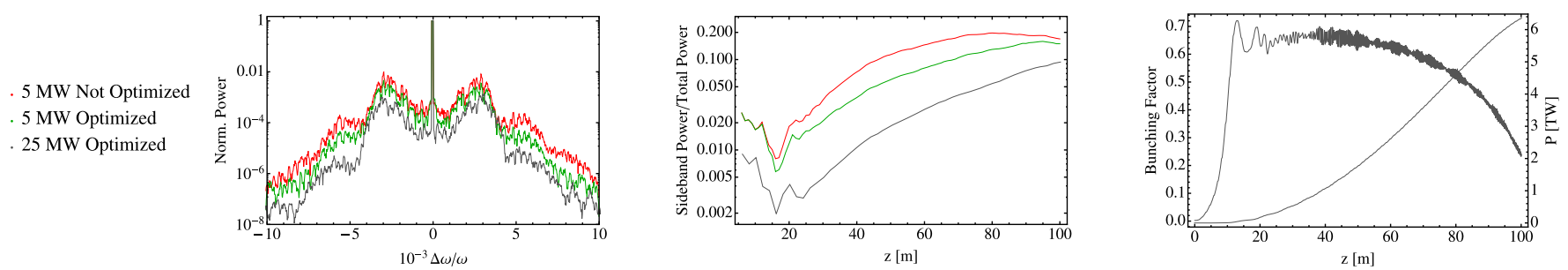

FIG. 6. (left) Spectrum at half the undulator length and fractional sideband power (middle) for the time independent optimized case and fully time dependent optimized for 5 and $25 \mathrm{MW}$ input seed power and $1.5 \mathrm{MeV}$ energy spread. Sideband power grows faster in the time independent optimized case, leading to particle detrapping and early saturation of the tapered FEL. (right) Bunching factor and power evolution for the optimal $25 \mathrm{MW}$ seed case.

the central wavelength by a quantity proportional to the synchrotron period:

$$
\lambda_{s^{\prime}} \approx \lambda_{s}\left[1 \pm \frac{\lambda_{w}}{L_{s y}}\right]=\lambda_{s}\left[1 \pm\left(\frac{a_{w} a_{s}}{1+a_{w}^{2}}\right)^{1 / 2}\right],
$$

where $L_{s y}$ is the synchrotron period. Once the sidebands are generated, the electron oscillations are driven by a multiple frequency ponderomotive potential. Therefore the equations of motion and Maxwell's equations for the electric field must be modified accordingly. An analysis of the simplest two frequency model shows that the coupled beam-radiation system is unstable, and that the sideband amplitude will grow from noise for any realistic electron distribution $[27,28]$. When the strength of the sidebands exceeds a critical level, electron motion becomes chaotic, leading to severe particle detrapping and a loss of amplification of the FEL signal [29]. Thus, as has been discussed by previous authors, suppressing the sideband instability is the key issue for tapered FEL designs [5], particularly those which are multiple synchrotron periods in length [30].

As is shown in Fig. 6, the time dependent optimized taper profile reduces sideband amplitude growth compared to the time dependent not optimized case obtained from the steady state taper profile. This results in a reduction in particle loss and a delayed taper saturation, both evidenced in the increased bunching factor and output power shown in Fig. 3. In the simple case of constant sideband and carrier amplitude, the diffusion coefficient caused by sideband excitations is proportional to the ratio of the power in the sidebands to the power in the FEL signal $D \propto C P_{s^{\prime}} / P_{s}$ with the coefficient $\mathrm{C}$ depending on the type of sideband spectrum [29]. As is also shown in Fig. 6, this is reduced in the time dependent optimized case. The peak power improves by 1 TW between the time dependent optimized and unoptimized cases, an efficiency increase of $2 \%$. Despite the dedicated time dependent optimization, we do not recover the single bucket extraction efficiency, unlike results previously reported in Ref. [31].

We note for completeness that in practice the sideband instability may start off with a stronger seed than just the shot noise in the electron beam current. This can arise for example from a microbunching induced spectral pedestal in the seed pulse [32] or fluctuations in the beam energy spread due to the spiky nature of the SASE process. While this has not been included in our study, in these cases the impact of time dependent effects is even more severe and a time independent tapering optimization will yield a solution even further from the optimum than the cases presented in our study.

\section{A. Sideband suppression}

In order to reach the single bucket extraction efficiency, we identify four separate solutions for further suppressing sideband growth which are currently being investigated. First, as was pointed out originally in Ref. [27] and demonstrated numerically by [33], increasing the electron beam energy spread in the last region of the undulator where the sideband amplitude is larger can also reduce the sideband growth.

The energy spread can be introduced by means of a magnetic delay line and the interaction of the beam with an external laser inside a short, few gain length undulator. The interaction should occur around the location of exponential saturation following the self-seeding monochromator. Depending on the flexibility of the undulator design, one may also obtain this additional energy spread by detuning a number of undulators around the exponential saturation location and allowing the beam to radiate spontaneously outside the FEL gain bandwidth [21].

Furthermore, a wavelength filter with corresponding delay line for the electrons could be placed in the tapered section in order to select a narrow bandwidth signal [30]. This should be done before the sideband power reaches the stochasticity threshold, beyond which the trapping efficiency is seriously degraded. Filtering out the sideband frequencies will result in a reduced bucket height and some initial particle detrapping. This should be compensated by a reduction in sideband induced detrapping between the filter and the end of the undulator, thus providing a lower limit to the bandwidth of the filter.

As a third solution, we note that the time independent efficiency can be achieved in a fully time dependent simulation by artificially removing the shot noise from 
the electron beam distribution after the SASE section. While this is not completely achievable in practice, electron beam shot noise suppression has been examined both theoretically and experimentally see Refs. [34-36]. Significant sideband reduction could be achieved if these schemes can be extended to hard x-ray wavelengths.

We note that the above three solutions present practical difficulties as an additional delay line aside from the selfseeding chicane is required to implement them in practice.

\section{B. Effect of the seed power}

Finally, one method to further minimize the effect of sideband growth that does not require additional delays is to increase the ratio of input seed power to equivalent shot noise power in the electron beam. We have analyzed the impact of this effect numerically by performing the same time dependent optimization described above with a $25 \mathrm{MW}$ input seed assuming the same initial energy spread of $\sigma_{E}=1.5 \mathrm{MeV}$ for comparison purposes. These parameters can be obtained by considering a fresh bunch system as discussed previously with a longer SASE section. While time independent tapering optimizations produce a peak power of 7.7 TW very similar to the $5 \mathrm{MW}$ seed case, time dependent optimizations have a much better performance, with a final output power of $6.3 \mathrm{TW}$. This is shown in Fig. 6, where we can see the peak radiation power still growing after $100 \mathrm{~m}$, and the bunching factor decaying slowly in the tapered section of the undulator. The mitigation of sideband-induced detrapping in this case is also evidenced by the fractional sideband power, which remains below $10 \%$ throughout the undulator.

\section{CONCLUSION}

In this paper we consider in detail the design of a high efficiency multi-TW hard x-ray FEL, a critical tool necessary for nonlinear $\mathrm{x}$-ray science and the 3D structural and dynamical study of single molecules. We analyze specifically the impact of time dependent effects on the efficiency of seeded tapered hard x-ray FELs by performing comparisons between time independent and time dependent tapering optimizations. To arrive at the efficiencies required to achieve TW peak powers in the shortest possible distance we propose a novel undulator design: helical, superconducting and with built-in transverse focusing. Using this undulator we perform a numerical optimization of the tapering profile in both time independent and fully time dependent simulations.

By comparing time independent and time dependent simulation results we demonstrate that the taper profile yielding the maximum power in time independent optimizations does not correspond to the optimal solution when time dependent effects such as slippage and electron beam shot noise are included in the simulation. We conclude that for a self-seeded system with an input energy spread of
3.1 MeV set by both the linac and the initial SASE section, the final output power increases from 2.7 TW with the time independent taper profile to $3.7 \mathrm{TW}$ with the profile obtained from dedicated time dependent scans.

We identify the sideband instability as the fundamental time dependent effect which is not taken into account in time independent optimizations, and limits the extraction efficiency by causing particle detrapping and saturation of the tapered FEL power. We have shown that with dedicated time dependent optimizations the fraction of energy deposited in the sidebands is reduced below the time independent case for the entire length of the undulator, limiting particle detrapping, delaying the taper saturation and increasing the efficiency of the FEL.

We have also outlined the importance of the trade-off in a self-seeded XFEL between energy spread and seed power at the entrance of the tapered undulator section. Using a "fresh bunch" with a smaller input energy spread we show that we can decrease particle detrapping, maintain a larger bunching factor and improve the overall performance for the same input seed power. We also propose using a fresh bunch system which can increase the input seed power by using a longer SASE section without affecting the input energy spread of the second bunch which lases in the seeded section. We have studied an optimal case with a $25 \mathrm{MW}$ seed and $1.5 \mathrm{MeV}$ energy spread and found that the output power reaches $6.3 \mathrm{TW}$ at the end of the undulator, an extraction efficiency of $12 \%$ very close to the time independent result of 7.7 TW.

While extending the simulation method of Ref. [7] to include time dependent effects significantly improves the performance of tapered XFELs, the current procedure is both time consuming and simulation intensive. With the enhanced understanding gained of the critical parameters limiting performance, such as the growth of the sideband instability, an improved algorithm can be developed which acts to directly suppress these effects. Such a scheme will be developed in future work.

\section{ACKNOWLEDGMENTS}

The authors would like to thank J. Duris, P. Musumeci, G. Marcus and A. Marinelli for useful discussions. We also acknowledge W. Fawley for sharing his expertise particularly with regards to the sideband instability. This work was supported by U.S. D.O.E. under Grant No. DE-SC0009983.

[1] P. Emma et al., First lasing and operation of angstromwavelength free-electron laser, Nat. Photonics 4, 641 (2010).

[2] T. Ishikaway et al., A compact x-ray free-electron laser emitting in the sub-angstrom region, Nat. Photonics 6, 540 (2012). 
[3] A. Aquila et al., The linac coherent light source single particle imaging road map, Struct. Dyn. 2, 041701 (2015).

[4] M. Fuchs et al., Anomalous nonlinear x-ray compton scattering, Nat. Phys. 11, 964 (2015).

[5] N. M. Kroll, P. L. Morton, and M. Rosenbluth, Freeelectron lasers with variable parameter wigglers, IEEE J. Quantum Electron. 17, 1436 (1981).

[6] W. M. Fawley, Z. Huang, K.-J. Kim, and N. A. Vinokurov, Tapered undulators for SASA FELs, Nucl. Instrum. Methods Phys. Res., Sect. A 483, 537 (2002).

[7] Y. Jiao, J. Wu, Y. Cai, A. W. Chao, W. M. Fawley, J. Frisch, Z. Huang, H.-D. Nuhn, C. Pellegrini, and S. Reiche, Modeling and multidimensional optimization of a tapered free electron laser, Phys. Rev. ST Accel. Beams 15, 050704 (2012).

[8] E. A. Schneidmiller and M. V. Yurkov, Optimization of a high efficiency free electron laser amplifier, Phys. Rev. ST Accel. Beams 18, 030705 (2015).

[9] A. Mak, F. Curbis, and S. Werin, Model-based optimization of tapered free-electron lasers, Phys. Rev. ST Accel. Beams 18, 040702 (2015).

[10] T. J. Orzechowski, B. R. Anderson, J. C. Clark, W. M. Fawley, A. C. Paul, D. Prosnitz, E. T. Scharlemann, S. M. Yarema, D. B. Hopkins, A. M. Sessler, and J. S. Wurtele, High-Efficiency Extraction of Microwave Radiation from a Tapered-Wiggler Free-Electron Laser, Phys. Rev. Lett. 57, 2172 (1986).

[11] D. Ratner et al., Fel gain length and taper measurements at LCLS, in Proceedings of the 31st International Free Electron Laser Conference (FEL 09), Liverpool, UK (STFC Daresbury Laboratory, Warrington, 2009), pp. 221-224.

[12] J. Duris, A. Murokh, and P. Musumeci, Tapering enhanced stimulated superradiant amplification, New J. Phys. 17, 063036 (2015).

[13] R. Bonifacio, C. Pellegrini, and L. Narducci, Collective instabilities and high-gain regime in a free electron laser, Opt. Commun. 50, 373 (1984).

[14] W. Smythe, Static and Dynamic Electricity (McGraw-Hill, New York, 1950).

[15] P. Emma et al., A plan for the development of superconducting undulator prototypes for LCLS-II and future fels, in FEL 2014 Conference Proceedings, Basel, Switzerland (JACoW, Basel, 2015), THA03.

[16] D. Prosnitz, A. Szoke, and V. K. Neil, High-gain, freeelectron laser amplifiers: Design considerations and simulation, Phys. Rev. A 24, 1436 (1981).

[17] A. Murokh et al., Results of the \{VISA $\{$ SASE $\{$ FEL experiment at $840 \mathrm{~nm}$, Nucl. Instrum. Methods Phys. Res., Sect. A 507, 417 (2003).

[18] S. Reiche and E. Prat, Growth rates and coherence properties of fodo-lattice based x-ray free electron lasers, in FEL 2012 Conference Proceedings, Nara, Japan (JACoW, Nara, 2013), p. MOOC02.

[19] C. Emma et al., Tapering studies for terawatt level x-ray FELs with a superconducting undulator, SLAC Report No. 16207, 2015.
[20] J. Amman et al., Demonstration of self-seeding in a hardX-ray free-electron laser, Nat. Photonics 6, 693 (2012).

[21] E. Saldin, E. Schneidmiller, and M. Yurkov, Calculation of energy diffusion in an electron beam due to quantum fluctuations of undulator radiation, Nucl. Instrum. Methods Phys. Res., Sect. A 381, 545 (1996).

[22] Z. Huang et al., Measurements of the linac coherent light source laser heater and its impact on the X-ray free-electron laser performance, Phys. Rev. ST Accel. Beams 13, 020703 (2010).

[23] A. Gover and E. Dyunin, Coherence limits of free electron lasers, IEEE J. Quantum Electron. 46, 1511 (2010).

[24] L. Giannessi, Harmonic generation and linewidth narrowing in seeded fels, in Proceedings of FEL2004 Conference (Comitato Conferenze Elettra, Trieste, Italy, 2004), pp. 37-40.

[25] S. Reiche, Genesis 1.3: A fully $3 \mathrm{~d}$ time-dependent fel simulation code, Nucl. Instrum. Methods Phys. Res., Sect. A 429, 243 (1999).

[26] C. Emma, J. Wu, K. Fang, S. Chen, S. Serkez, and C. Pellegrini, Terawatt $\mathrm{X}$-ray free-electron-laser optimization by transverse electron distribution shaping, Phys. Rev. ST Accel. Beams 17, 110701 (2014).

[27] N. R. M. Kroll, Introduction to the physics of the free electron laser, in Free Electron Generators of Coherent Radiation, edited by S. Jacobs, H. Pilloff, M. Sargent, M. Scully, and R. Spitzer (Springer, Heidelberg, 1979), pp. 147-174.

[28] S. Riyopoulos and C.M. Tang, The structure of the sideband spectrum in free electron lasers, Phys. Fluids 31, 1708 (1988).

[29] S. Riyopoulos and C. M. Tang, Chaotic electron motion caused by sidebands in free electron lasers, Phys. Fluids 31, 3387 (1988).

[30] D. C. Quimby, J. M. Slater, and M. Wilcoxon, Sideband suppression in free electron lasers with multiple synchrotron periods, IEEE J. Quantum Electron. 21, 979 (1985).

[31] B. Hafizi, A. Ting, P. Sprangle, and C. M. Tang, Development of sidebands in tapered and untapered free electron lasers, Phys. Rev. A 38, 197 (1988).

[32] Z. Zhang et al., Microbunching-instability-induced sidebands in a seeded free-electron laser, in FEL 2015 Conference Proceedings, Daejeon, Korea (to be published).

[33] J. Goldstein and W. Colson, Control of optimal pulse modulation due to the sideband instability in free electron lasers, in Proceedings of the International Conference on Lasers, New Orleans, LA (STS Press, New Orleans, 1983), pp. 218-225.

[34] E. Dyunin and A. Gover, The general velocity and current modulation linear transfer matrix of $\{$ FEL $\}$ and control over $\{\mathrm{SASE}\}$ power in the collective regime, Nucl. Instrum. Methods Phys. Res., Sect. A 593, 49 (2008).

[35] A. Nause, E. Dyunin, and A. Gover, Short wavelength limits of current shot noise suppression, Phys. Plasmas 21, 083114 (2014).

[36] D. Ratner, Z. Huang, and G. Stupakov, Analysis of shot noise suppression for electron beams, Phys. Rev. ST Accel. Beams 14, 060710 (2011). 